\title{
CONFIRMACIÓN DE LA PRESENCIA DE LA SERPIENTE PITUOPHIS CATENIFER (BLAINVILLE, 1835) EN EL ESTADO DE HIDALGO, MÉXICO
}

\section{CONFIRMATION OF THE PRESENCE OF SERPENT PITUOPHIS CATENIFER (BLAINVILLE, 1835) IN THE STATE OF HIDALGO, MEXICO}

\author{
LeONARdo Fernández-Badillo ${ }^{1,2 *}$, GonZalo HernándeZ-HeRnándeZ ${ }^{3}$, Raúl VAlencia-Herverth ${ }^{3}$, \\ IRENE GOYENECHEA ${ }^{4}$ Y ClaUdio A. MENDOZA PAZ ${ }^{3}$ \\ ${ }^{1}$ Centro de Investigaciones Biológicas (CIB), Universidad Autónoma del Estado de Hidalgo, Ciudad del conocimiento, km 4.5 carretera Pachuca- \\ Tulancingo, Col. Carboneras, 42181 Mineral de la Reforma, Hidalgo. \\ ${ }^{2}$ Predio Intensivo de Manejo de Vida Silvestre X-Plora Reptilia, carretera México-Tampico s/n, Pilas y granadas, 43350, Metztitlán, Hidalgo, México. \\ ${ }^{3}$ Instituto Tecnológico de Huejutla, Tecnológico Nacional de México, carretera Huejutla-Chalahuiyapa, km 5.5.Huejutla de Reyes, Hidalgo. \\ ${ }^{4}$ Laboratorio de Sistemática Molecular. Centro de Investigaciones Biológicas (CIB), Universidad Autónoma del Estado de Hidalgo, Ciudad del \\ conocimiento, km 4.5 carretera Pachuca-Tulancingo, Col. Carboneras, 42181 Mineral de la Reforma, Hidalgo. \\ *Correspondence:fernandezbadillo8o@gmail.com
}

Abstract.- Recent studies on Hidalgo's herpetofauna only consider one species of the genus Pituophis, $P$. deppei, to be present in the state. However, previous records of $P$. catenifer in Hidalgo have not been taken into account. In addition, fieldwork conducted in northern Hidalgo produced four additional individuals of P. catenifer from the municipalities of San Felipe Orizatlán and Huejutla de Reyes, corroborating the presence of this species in Hidalgo.

Keywords.- Corroboration, species, genus, herpetofauna.

Resumen.- Los estudios recientes acerca de la herpetofauna de Hidalgo únicamente consideran a una especie del género Pituophis presente en la entidad (P. deppei). Sin embargo, existen registros previos de $P$. catenifer que no han sido tomados en cuenta. Por otro lado, a partir del trabajo de campo realizado al norte del estado, se registraron otros cuatro ejemplares de P. catenifer en los municipios de San Felipe Orizatlán y Huejutla de Reyes, lo que corrobora la presencia de esta especie en Hidalgo.

Palabras clave.- Corroboración, especie, género, herpetofauna.

Pituophis catenifer es un colúbrido que se encuentra ampliamente distribuido en el norte de México y sur de los Estados Unidos de Norteamérica (Bryson et al., 2011; Heimes, 2016); Heimes (2016), muestra un mapa en el que se observa que su distribución abarca hasta la parte norte de Hidalgo, sin embargo, no menciona su presencia para este estado en el apartado de distribución de la especie. Por otro lado, los estudios recientes sobre herpetofauna de Hidalgo (Ramírez-Bautista et al., 2014; Lemos-Espinal y Dixon, 2016; Manríquez-Morán et al., 2017) o específicamente sobre serpientes de la entidad (Fernández-Badillo et al., 2017), consideran únicamente a una especie del género Pituophis presente en Hidalgo: P. deppei. Sin embargo, Bryson et al. (2011) incluyeron en su análisis tejido de un par de organismos de Pituophis catenifer de la localidad de Chalahuiyapa, municipio de Huejutla de Reyes (Las Vegas Tissue Collection, número de voucher 10659, 10660), sin que hasta la fecha se tuvieran otros registros publicados que confirmaran la presencia de esta especie en Hidalgo. En este trabajo se presentan cuatro localidades nuevas para la especie en Hidalgo, lo que corrobora la información presentada por Bryson et al. (2011) e incrementa el conocimiento de la distribución de la especie en Hidalgo.

A partir de trabajo de campo realizado en los municipios de San Felipe Orizatlán y Huejutla de Reyes, se obtuvieron registros de la serpiente Pituophis catenifer en distintas localidades, cuyas fotografías, así como un ejemplar recolectado se depositaron en la colección herpetológica del Centro de Investigaciones Biológicas de la Universidad Autónoma del Estado de Hidalgo (CH-CIB). Para el caso de San Felipe Orizatlán, se registraron ejemplares en tres localidades: Valle Verde $\left(21.180257^{\circ} \mathrm{N}\right.$, $98.588649^{\circ} \mathrm{O}, 206$ m elevación; Fig. 1), 10 de mayo de 2016, un ejemplar adulto (CH-CIB 114; Fig. 2a); Zacayahual $\left(21.175684^{\circ} \mathrm{N}\right.$, 98.607941 ${ }^{\circ} \mathrm{O}, 155 \mathrm{~m}$ elevación; Fig. 1), 17 de agosto de 2016, un ejemplar adulto recolectado (CH-CIB 5028, Fig. 2b) y Cececamel 


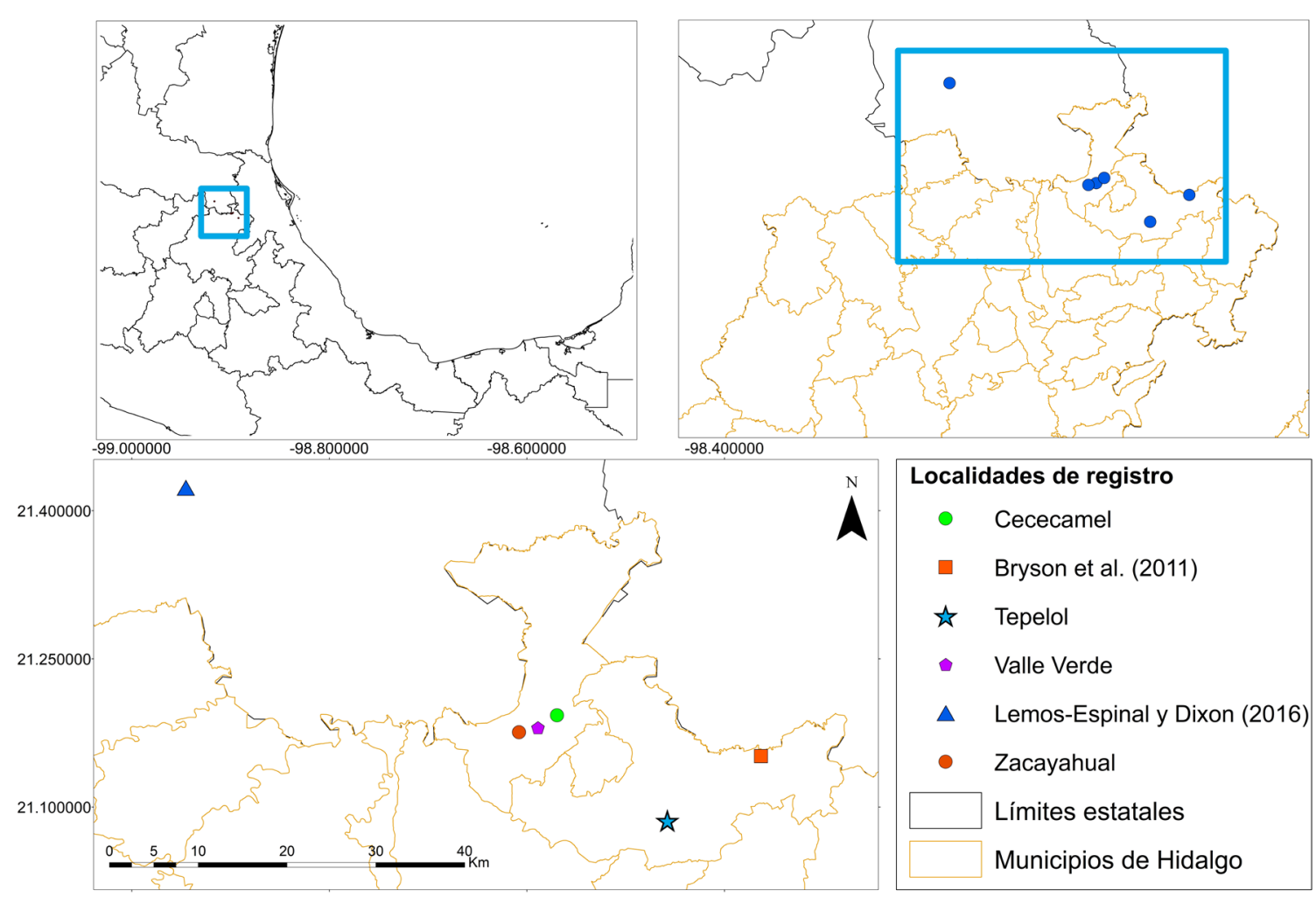

Figure 1. Map that shows the locality records of Pituophis catenifer in the state of Hidalgo, as well as its closest locality, located in Xilitla, San Luis Potosi, Mexico.

Figura 1. Mapa que muestra los sitios de registro de Pituophis catenifer en el estado de Hidalgo, así como la localidad más cercana a estos, ubicada en Xilitla, San Luis Potosí, México.

(21.192752 $\mathrm{N}, 98.569455^{\circ} \mathrm{O}, 210$ m elevación; Fig. 1), 12 de mayo de 2017, un ejemplar juvenil (CH-CIB 99; Fig. 2c). En el municipio de Huejutla de Reyes, se registró en la localidad de Tepeolol $\left(21.086111^{\circ} \mathrm{N}, 98.457778^{\circ} \mathrm{O}, 259 \mathrm{~m}\right.$ elevación; Fig. 1), 18 de octubre de 2017, un ejemplar juvenil (CH-CIB 115; Fig. 2d). El colector para las primeras tres localidades fue Gonzalo Hernández Hernández y Claudio A. Mendoza Paz para la última.

Los ejemplares de las localidades de Valle Verde y Zacayahual fueron encontrados por pobladores locales, quienes avisaron de la presencia de las serpientes y afirmaron haberlas matado porque consideraron que se trataba de serpientes venenosas. Sin embargo, se desconoce si en general todos los habitantes confunden a esta especie con alguna serpiente venenosa. Debido al grado de descomposición del ejemplar de Valle Verde, este no fue recolectado y únicamente se le tomó una fotografía. Las serpientes de Cececamel y Tepeolol fueron encontradas vivas, se fotografiaron y liberaron en el sitio de la observación. Todas las localidades de registro fueron zonas perturbadas (potreros, cultivos) rodeadas de vegetación nativa (bosque tropical perennifolio).
Todos los ejemplares encontrados en este trabajo coinciden con la descripción de la especie mencionada por Lemos-Espinal y Dixon (2013), cuya característica principal es la presencia de cuatro escamas prefrontales, a diferencia de lo que ocurre en P. deppei, la cual únicamente presenta dos. Este carácter puede diagnosticarse a simple vista, al observar el dorso de la cabeza de la serpiente (Fig.3). Los ejemplares del municipio de San Felipe Orizatlán, llenan el vacío en la distribución conocida de la especie entre los registros en la localidad de Xilitla en el estado de San Luis Potosí (Lemos-Espinal \& Dixon, 2013) y los registros previos para Hidalgo mencionados por Bryson et al. (2011).

La presencia de esta especie en una zona de bosque tropical perennifolio es poco usual, y como tal no ha sido mencionada explícitamente en otros estudios, sin embargo, existen otros registros que concuerdan con este tipo de vegetación, como por ejemplo el de Dixon y Lemos-Espinal (2013) para la localidad de Xilitla, San Luis Potosí. Por otro lado, Pérez-Higareda (1981) menciona los registros de tres ejemplares de la especie cercanos a la ciudad de Veracruz, en las localidades de Boca del Río (13 km al sur del puerto de Veracruz), El Bajo del Ingeniero y San Julián (15 


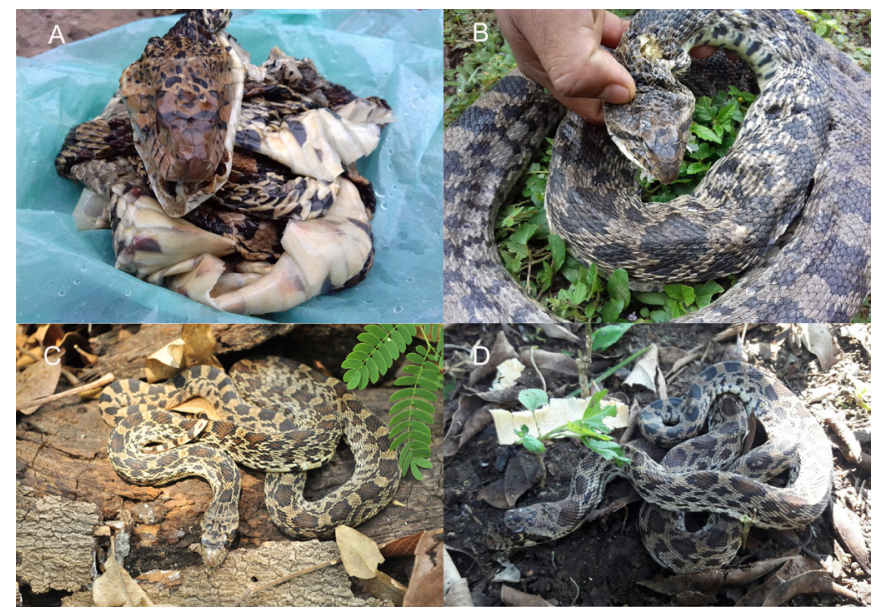

Figure 2. Pictures of specimens of Pituophis catenifer registered in Hidalgo, México. Localities: a) Valle Verde (CH-CIB 113); b) Zacayahual (CH-CIB 5028); C) Cececamel (CH-CIB 99), d) Tepeolol (CH-CIB 115). Photographes by: a) Gonzalo Hernández-Hernández, b y c) Leonardo Fernández-Badillo y d) Claudio Mendoza-Paz.

Figura 2. Fotografías de los ejemplares de Pituophis catenifer registrados en Hidalgo, México. Localidades: a) Valle Verde (CH-CIB 113); b) Zacayahual (CH-CIB 5028); c) Cececamel (CH-CIB 99), d) Tepeolol (CH-CIB 115). Fotografías: a) Gonzalo Hernández-Hernández, b y c) Leonardo Fernández-Badillo y d) Claudio Mendoza-Paz.

$\mathrm{km}$ al norte del puerto de Veracruz), los cuales, por su ubicación, concuerdan con un área de bosque tropical perennifolio, aunque el autor sugiere que probablemente son ejemplares introducidos. Sin embargo, considerando la información aquí presentada, también permite llenar el vacío en la distribución de $P$. catenifer, entre los registros al sur de San Luis Potosí y los registros cercanos a la Ciudad de Veracruz.
Bryson et al. (2011) mencionan que la distribución conocida de $P$. catenifer está conformada por tres filogrupos que presentan divergencias relativamente recientes, lo que sugiere que la especie reconocida como $P$. catenifer debe ser más bien un complejo de especies. Por lo tanto, el tipo de hábitat podría ser otra característica que apoye las diferencias en los filogrupos detectados por Bryson et al. (2011).

Agradecimientos.- A los proyectos "Diversidad Biológica del Estado de Hidalgo (tercera etapa) FOMIX-CONACyTHGO-2012-191908” y SEP-CONACyT Ciencia Básica 222632, por el apoyo logístico y económico para el trabajo de campo. A la SEMARNAT por la autorización brindada para la recolecta de los ejemplares, mediante el número de oficio SEMARNATFAUT-0052.

\section{LITERATURA CITADA}

Bryson, R.W. Jr., U.O. García-Vázquez \& B.R. Riddle. 2011 Phylogeography of Middle American gophersnakes: mixed responses to biogeographical barriers across the Mexican Transition Zone. Journal of Biogeography 38:1570-1584.

Fernández-Badillo, L., N. Morales-Capellán, C.R. Olvera-Olvera, G. Montiel-Canales \& I. Goyenechea. 2017. Guía de las serpientes del estado de Hidalgo. Pachuca de Soto, Hidalgo. Universidad Autónoma del Estado de Hidalgo, Pachuca de Soto, Hidalgo.

Heimes, P. 2016. Herpetofauna Mexicana Vol.1. Snakes of Mexico. Edition Chimaira. Frankfurt am Main, Germany.
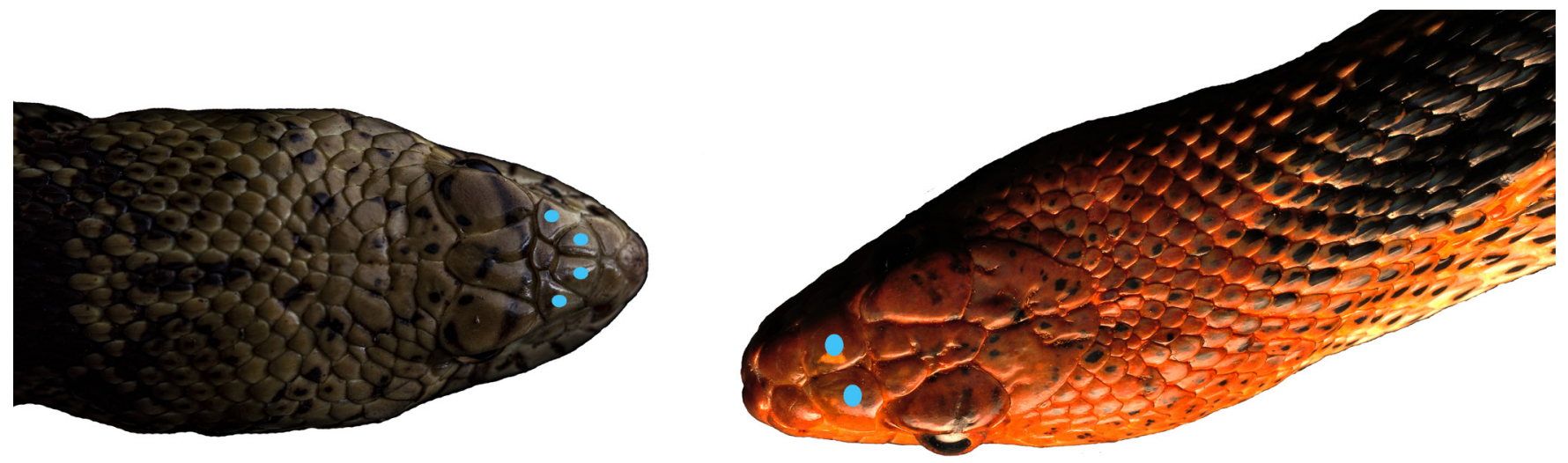

Figure 3. Pictures showing the dorsal portion of the head of P.catenifer (left) y P. deppei (right), the blue dots indicate the prefrontal scales.

Figura 3. Fotografías que muestran la parte dorsal de la cabeza de P.catenifer (a la izquierda) y P. deppei (a la derecha), con puntos azules se indican las escamas prefrontales. 
Lemos-Espinal, J.A. \& J.R. Dixon. 2013. Amphibians and reptiles of San Luis Potosí. Eagle Mountain, Eagle Mountain Publishing L.C. Utah.

Lemos-Espinal, J.A. \& J.R. Dixon. 2016. Anfibios y reptiles de Hidalgo, México. Comisión Nacional para el Conocimiento y Uso de la Biodiversidad. México, D.F.

Manríquez-Morán, N.L., J.M. Castillo-Cerón, I. Goyenechea, R. Cruz-Elizalde, U. Hernández-Salinas, D. Lara-Tufiño, L.M. Badillo-Saldaña, C.S Berriozabal-Islas \& A. Ramírez-Bautista. 2017. Riqueza y diversidad de saurópsidos (no aves) del estado de Hidalgo Pp. 536-562. En A. Ramírez-Bautista, A. Sánchez-
González, G. Sánchez-Rojas, y M. C. Cuevas-Cardona (Eds.), Biodiversidad del Estado de Hidalgo. Tomo II. UAEH/CONACyT, Pachuca.

Pérez-Higareda, G., \& H. Smith. 1981. Ofidiofauna de Veracruz. Análisis taxonómico y zoogeográfico. Publicaciones especiales Instituto de Biología 7, UNAM, México.

Ramírez-Bautista, A., U. Hernández-Salinas, R. Cruz-Elizalde, C.S. Berriozabal-Islas, D. Lara-Tufiño, I. Goyenechea MayerGoyenechea \& J.M. Castillo-Cerón. 2014. Los Anfibios y Reptiles del Estado de Hidalgo, México: Diversidad, biogeografía y conservación. Sociedad Herpetológica Mexicana, Pachuca. 